\title{
A Diagrammatic Inference System for the Web
}

\author{
Michael Wollowski \\ Rose-Hulman Institute of Technology \\ 5500 Wabash Ave. \\ Terre Haute, IN 47803, USA \\ 001-812-877-8650 \\ wollowski@rose-hulman.edu
}

\author{
Peter $\mathrm{Nei}^{1}$ \\ Beckman Coultier., PDC \\ 7451 Winton Drive \\ Indianapolis, IN 46268, USA \\ 001-317-808-4382 \\ peter.nei@sagian.com
}

\author{
Chris Barrell $^{1}$ \\ Logikos, Inc. \\ 2914 Independence Drive \\ Fort Wayne, IN 46808 \\ 001-260-373-1387 \\ cbarrell@logikos.com
}

\begin{abstract}
We developed a diagrammatic inference system for the World Wide Web. Our system enables the creation of diagrams such that the information contained in them can be searched and inference can be performed on it. We developed an XMLSchema for bar, line, and pie charts. Based on it, we developed software that transforms a corresponding XML file into an SVG image, which in turn is rendered by the client as an image. Additionally, we developed a search engine which enables a user to find information explicitly contained in the XML file, and as such in the image. Furthermore, we developed an inference engine which enables a user to locate information that is implicitly contained in the image.
\end{abstract}

\section{Categories and Subject Descriptors}

H.3.5 [Online Information Services]: Web-based services

\section{General Terms}

Human Factors, Standardization.

\section{Keywords}

Search, diagrams, searchable diagrams, inference system, XML.

\section{INTRODUCTION}

We developed a process for presenting diagrammatic information on the World Wide Web while making that information available to a search and inference engine. If done right, this makes the information easier to read for people [2]. However, information presented in an image file is not accessible to search engines. While a search engine may be able to use the image tags and surrounding text to make an educated guess about what an image represents, in the case of diagrams (as compared to photos) it is particularly desirable to be able to search and reason with the information contained within the image.

Diagrammatic inference systems are special-purpose systems. They differ from first-order logic in that they are custom-fit for the domain for which they are intended. This means that they are limited to that domain, but they are usually complete $[3,4,5]$. See [1] for an encompassing treatment of these issues.

\section{AN EXAMPLE}

In the following example, we show the format of an XML document in our system and how it relates to the rendered image.

Copyright is held by the author/owner(s).

$W W W$ 2004, May 17-22, 2004, New York, NY USA.
Figure 1 contains the XML file and figure 2 the rendered image. Data is boldfaced.

The top level element is chart and contains a single attribute type with which to specify the chart type, which can be a bar-, line-, or pie-chart. In this example, we show a bar chart. The chart's title is contained in the title element and is displayed above the chart proper. The title not only assists the human viewer in identifying the kind of information presented, the search engine will also rely heavily on the information in it.

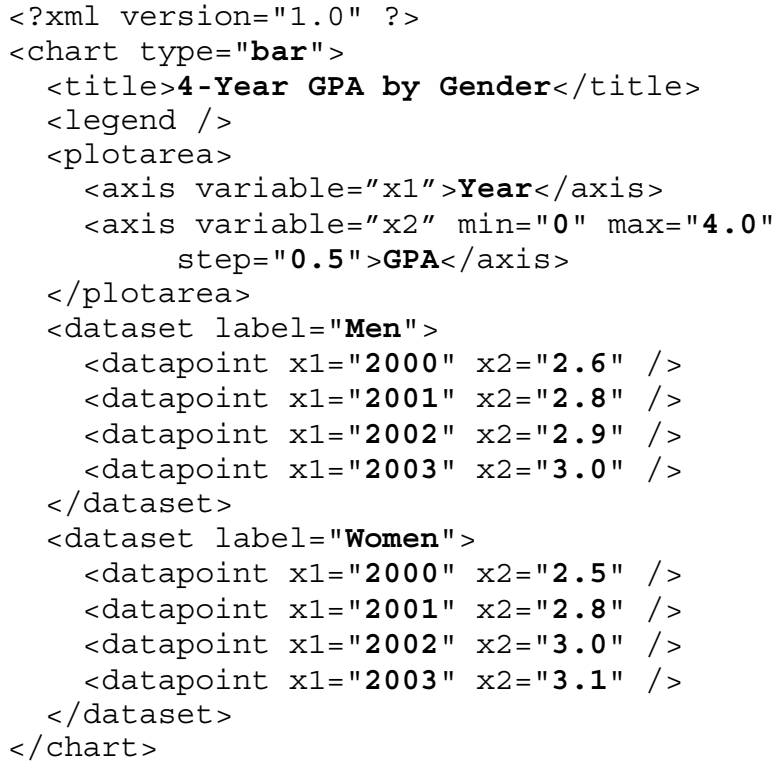

Figure 1. A sample XML file for a bar chart.

A legend element enables the user to specify (by its presence or absence) whether a legend should be generated in the resulting image. The actual label information is pulled from the dataset elements, as described below.

The information enclosed in the plotarea element is of technical as well as semantic nature. Currently, our system only renders two dimensional charts, designated by variable attributes with the values "x1" and "x2". The text contained within an axis element is used to label it. For a bar chart, the x2axis specifies a starting and ending value (min and max attribute) as well as a gradation for the vertical axis (step attribute). In contrast, the horizontal $\mathrm{x} 1$-axis does not contain these attributes and is instead divided based on the string data present in the $\mathrm{x} 1$

\footnotetext{
1 This work was done while the authors were at Rose-Hulman Institute of Technology.
} 
attributes of datapoint elements. For a line chart, both axes contain numerical data and hence would look like the $\mathrm{x} 2$-axis of the example. In contrast, a pie chart has no axes and has no need of a plotarea element.

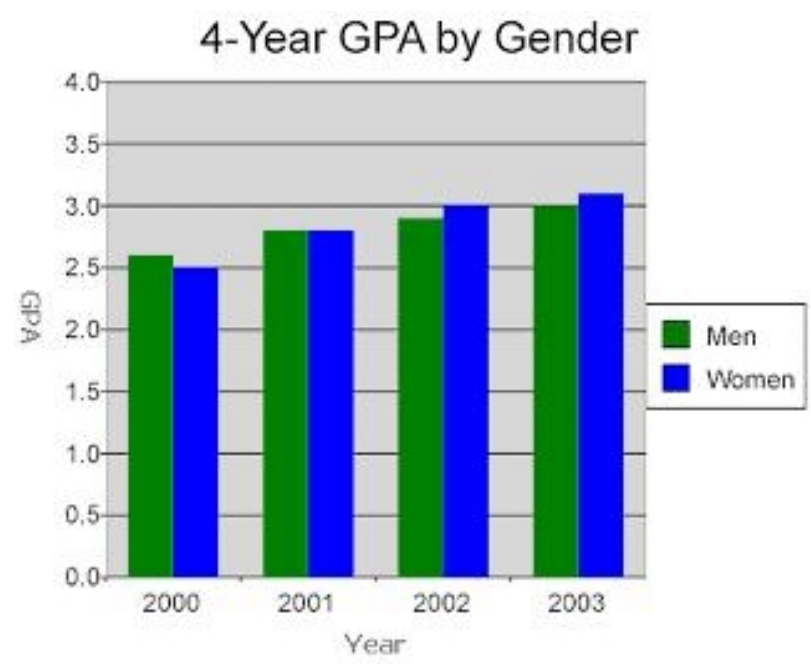

Figure 2. The rendered image of the file from figure 1.

Each set of data is enclosed in a separate dataset element. This element has a label attribute identifying the dataset in the legend. In our example, we have data for men and for women. A dataset consists of datapoint elements. Every datapoint has two attributes identifying the values across the horizontal axis (x1) and the vertical axis (x2). Data points are represented by bars in a bar chart and through a connected line in a line chart. In a pie chart, each dataset represents a slice of the pie, and instead of containing datapoint elements, it has a second attribute, value.

\section{DIAGRAMMATIC SEARCH AND INFERENCE ENGINE}

In order for a search engine to return meaningful results, we designed a special-purpose search engine which is tailor made for our XML format. See [5] for more information on special-purpose XML search engines.

To get a sense of how to interpret the data of charts in our system, we will rewrite a datapoint. First, we substitute in the contents of the title element and the value of label attribute from its surrounding dataset element. We then substitute the $\mathrm{x} 1$ and $\mathrm{x} 2$ attributes with the contents of their corresponding axis elements. The resulting first datapoint of the dataset for men looks as follows:

$$
<4 \text {-Year GPA by Gender Men "Year = 2000" "GPA = 2.6"/> }
$$

Each datapoint now contains all information pertaining to it. At this point, we can search each datapoint individually and display all that satisfy a given query. Suppose that the user enters the keyword "women". The results of this query are displayed in figure 3 . In it, we return all datapoints, as modified above, which contain this keyword. The results are presented in a nested fashion, so as to avoid repetition of information. Furthermore, a link to the source XML document is presented.

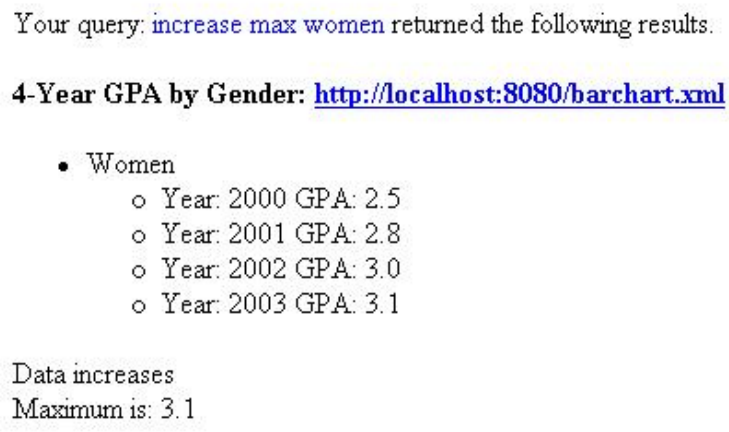

Figure 3. The results of a query requesting inference.

A search engine enables a user to find information that is explicitly contained in a document. An inference engine enables a user to also search for information that is implicitly contained in the document. For example, the highest GPA achieved by women is 3.1. Furthermore, the GPA went up each year. Those two results are indicated in the search displayed in figure 3. They are two pieces of information that people looking at bar-charts are very interested in. We implemented those two inference rules, as well as rules for minimum, average, median, decreasing and those that compare datapoints to a given values. We feel that those predicates are central to charts of our system, and are sufficient for now.

\section{CONCLUSIONS}

Our system of storing chart information in XML files on the web demonstrates a general process by which diagrammatic information can be stored on a web server in sentential format without losing the preferred visual presentation of the information to the user. The primary benefit of this process is that what the user perceives as an image is in fact a text file that can be indexed by a typical search engine spider. The choice of an XML format reaps the additional benefit of having structured metadata, as demonstrated in the design of our diagrammatic search and inference engine.

\section{REFERENCES}

[1] Barwise, Jon and Etchemendy, John. Visual information and valid reasoning. in: Visualization in Mathematics, Zimmerman and Cunningham (eds.), Mathematical Association of America, Washington, D.C., 1990.

[2] Glasgow, Janice, Narayanan, N.Hari, and Chandrasekaran, B. (eds.). Diagrammatic Reasoning. AAAI/MIT Press, Menlo Park, CA, 1995.

[3] Hammer, Eric. Logic and Visual Information. CSLI Press, Stanford, CA, 1995.

[4] Allwein, Gerard and Barwise, Jon (eds.). Logical Reasoning with Diagrams, Oxford University Press, 1996.

[5] Wollowski, Michael and Signorelli, Robert. Towards a General-Purpose XML Search Engine. Presentation at the 8th Search Engine Meeting, Boston, MA, 2000. 